\title{
OPEN Modeling cross-field demagnetization of superconducting stacks and bulks for up to 100 tapes and 2 million cycles
}

\author{
Anang Dadhich \& Enric Pardo
}

Superconducting stacks and bulks can act as very strong magnets (more than $17 \mathrm{~T}$ ), but they lose their magnetization in the presence of alternating (or ripple) transverse magnetic fields, due to the dynamic magneto-resistance. This demagnetization is a major concern for applications requiring high run times, such as motors and generators, where ripple fields are of high amplitude and frequency. We have developed a numerical model based on dynamic magneto-resistance that is much faster than the conventional Power-Law-resistivity model, enabling us to simulate high number of cycles with the same accuracy. We simulate demagnetization behavior of superconducting stacks made of 10-100 tapes for up to 2 million cycles of applied ripple field. We found that for high number of cycles, the trapped field reaches non-zero stationary values for both superconducting bulks and stacks; as long as the ripple field amplitudes are below the parallel penetration field, being determined by the penetration field for a single tape in stacks. Bulks keep substantial stationary values for much higher ripple field amplitudes than the stacks, being relevant for high number of cycles. However, for low number of cycles, stacks lose much less magnetization as compared to bulks.

High temperature superconductors (HTS) are very promising for next-generation electric devices, such as largefield or large-bore magnets, power-transmission cables, fault-current limiters, transformers, generators, and motors. In rotating machines (motors and generators), HTS enable substantial reductions in weight, size and energy loss compared to their conventional counterparts of the same power and torque. Some examples are wind generators ${ }^{1-4}$, motors for ship propulsion ${ }^{5-9}$, and linear motors for high-speed conventional or levitating trains ${ }^{10-14}$ or Hyperloop ${ }^{15}$. Superconducting motors are also proposed to be used for hybrid distributed electric propulsion for aircraft ${ }^{8,16-19}$, which can help in reducing $\mathrm{CO}_{2}$ and $\mathrm{NO}_{x}$ emissions by $75 \%$, fuel burnt by $70 \%$ and noise by $71 \mathrm{db}^{8}$. REBCO $\left(R E B a_{2} C_{3} \mathrm{O}_{7-x}\right.$, where the rare-earth $R E$ is usually Y or Gd) HTS bulks and stacks of tapes can trap high amounts of magnetic fields, with current record being $17.6 \mathrm{~T}$ for bulks ${ }^{20}$ and 17.7 $\mathrm{T}$ for stacks ${ }^{21}$, and can be a viable option for building rotors in HTS motors, instead of coils (using stacks ${ }^{19,22-24}$ or bulks ${ }^{7,8}$ ). The main benefits of superconducting stacks or bulks over coils are the compactness of size, sturdy material and geometry, and exclusion of current leads which accounts for substantial reduction of heat load ${ }^{25}$. In addition, stacks of tapes and bulks can also generate the levitation force in levitating trains and flyweels, see s-26 $^{26}$ for bulks and $\mathrm{d}^{29-32}$ for stacks. Finally, stacks of tapes can also enable compact magnets, such as those for Nuclear Magnetic Resonance ${ }^{33}$. Stacks present several advantages compared to bulks such as higher engineering current densities, longer lengths (up to hundreds of meters), higher engineering current densities, homogeneity, flexibility, and enhanced mechanical and thermal properties thanks to its layered structure ${ }^{34}$.

Although stacks and bulks enable to design very powerful superconducting magnets, they lose some magnetization in the presence of alternating (or AC) transverse magnetic fields, and hence the application is left with relatively low power output ${ }^{35-38}$. The cause for this demagnetization is a well known dissipative effect called dynamic magneto-resistance ${ }^{39}$.

On application of a transverse AC magnetic field above a certain threshold field to a superconducting tape, a direct current (DC) voltage appears that opposes the present DC transport current. In motors and generators, these transverse AC fields are caused by the background ripple fields, mostly originated by the stator. Then, 
dynamic magneto-resistance or "effective resistance" occurs, which causes the decay of the critical currents and, subsequently, demagnetization of the tape $\mathrm{e}^{40}$. According to Brandt et al. ${ }^{39}$, this relaxation is due to the "walkingmotion" of vortices in the thin tape. However, the dynamic magneto-resistance can also be explained by the Critical State Model $^{41}$, and hence the presence of vortices is not strictly required ${ }^{42}$. Below threshold field, $B_{t h}$, a shielded region of "frozen-flux" exists within the center, such that no change in field is experienced by the DC current flowing in this shielded region, and hence the dynamic magneto-resistance is zero because there is no flux crossing the superconductor ${ }^{41}$. This resistance is also the cause of an additional power loss that is required to be considered when calculating the total loss in superconductor, which also includes the magnetization loss ${ }^{40}$.

Cross-field demagnetization depends on various parameters, such as the tape geometry and the applied ripple field properties. It increases with the applied field amplitude and frequency, tape thickness, and tape length, and decreases with the critical current density and the width of the tape ${ }^{43-45}$. The geometry of the HTS stacks also plays an important role. Firstly, the cross-field demagnetization rate is roughly inversely proportional to the number of tapes ${ }^{36,44,45}$. Secondly, ferromagnetic materials can also decrease the demagnetization rate, either by intercalating layers between the tapes ${ }^{36}$, or by placing the stack between ferromagnetic plates ${ }^{45}$. More sophisticated structures, such as buried C-shaped stacks in the rotor iron, are also expected to present low cross-field demagnetization $^{46}$. In the presence of high background ripple field amplitudes and frequencies ranging in a few thousand $\mathrm{Hz}$, as is the case of fully superconducting motors in aviation, the value of dynamic resistance is relatively high, and thus there is a fast decay of magnetization in both superconducting stacks ${ }^{47}$, and bulks ${ }^{36,48-51}$. Thus, understanding cross field demagnetization (caused by dynamic magneto-resistance) for superconducting stacks and bulks is an issue of utmost importance.

Brandt et al. ${ }^{39}$ also show that for applied ripple fields below threshold field, the trapped field for a single tape reaches an asymptotic value after high number of cycles that remains indefinitely. It is important to know if the same behavior exists for bulks and stacks, i.e. under what conditions there will be a non-zero asymptotic trapped field output, if any, and its magnitude. Unfortunately, it has been a very tough and time-consuming task to numerically model high number of cycles with high mesh and field accuracy. Therefore, it is the goal of this paper to model cross field demagnetization for high number of cycles (up to millions) using a unique approach involving dynamic magneto-resistance. Although it has not been currently possible to model stacks involving more than 20 tapes, this paper presents results for up to 100-tape thick stacks, as realistic stacks of tapes often include between 50 and 100 HTS tapes ${ }^{46}$. A detailed comparison of superconducting stacks and bulks facing the issue of demagnetization is also required, which this paper achieves.

The structure of this paper is the following. Firstly, we present the numerical model developed by our team using dynamic magneto-resistance (DMR model), and give a brief discussion on its benefits. Next, the DMR model is benchmarked with traditional models. Then, the results are presented for a commercial single tape, and stacks involving 10 and 100 tapes, for high number of cycles. We conclude our paper by comparing the potential benefits of stacks and bulks over each other for different cases related to cross field demagnetization.

\section{Modeling method}

We use our in-house 2D Minimum Electro Magnetic Entropy Production (MEMEP) variational method ${ }^{52,53}$ to model the demagnetization behavior of superconducting stacks in the presence of cross-fields. In $2 \mathrm{D}$, the $\mathbf{J}$ -formulation used in MEMEP is much faster than conventional finite element methods (FEM), since we do not have to consider surrounding air into the mesh, which saves many degrees of freedom ${ }^{54}$. This method has been extensively benchmarked against conventional $H$-formulation method (COMSOL) and experiments, and have produced excellent results for the given case $\mathrm{e}^{38,49,52,55}$. We also have seen that, whereas the $3 \mathrm{D}$ method is important for short samples, the $2 \mathrm{D}$ cross-sectional simplification can be used when the tape length is 3 times or more than the tape width, producing practically the same results as the full $3 \mathrm{D}$ method, but with much less computing time ${ }^{45}$.

The MEMEP method can take any $E(J)$ relation into account, and in particular those described in the following section. For the primary results of this paper, we use an effective constitutive relation for the dynamic magneto-resistance (DMR model), as described below.

Constitutive relations of the superconductor. For our $2 \mathrm{D}$ cross-sectional approach, where the tapes are infinitely long in the $z$ direction, both electric field, $\mathbf{E}$, and current density, $\mathbf{J}$, follow $\mathbf{E}=E \mathbf{e}_{z}$ and $\mathbf{J}=J \mathbf{e}_{z}$, being $\mathbf{e}_{z}$ the unit vector along the $z$ axis. In this article, we refer to both the magnetic flux density, $\mathbf{B}$, and magnetic field, $\mathbf{H}$, as "magnetic field" because we do not take magnetic materials into account, and hence $\mathbf{B}=\mu_{0} \mathbf{H}$. For ease of terminology, we refer to the magnetic field as simply "field".

Power-law E(J) relation. A usual choice for modeling superconductors is to use the following power-law $\mathrm{E}(\mathrm{J})$ relation, which experimentally agrees with voltage-current measurements for $J$ close to $J_{c}$. We use this model for benchmarking the proposed DMR model.

$$
E(J)=E_{c}\left(\frac{|J|}{J_{c}}\right)^{n} \frac{J}{|J|}
$$

where, $E, J, E_{c}, J_{c}$, and $n$ are the electric field, current density, voltage criterion $\left(10^{-4} \mathrm{~V} / \mathrm{m}\right.$ in our case), critical current density, and power law exponent, respectively.

Critical state model. The critical state model (CSM) postulates that any electromotive force induces current with critical current density, $J_{c}$, in a superconductor ${ }^{56}$. For infinitely long geometries of finite thickness, $|J|$ only takes 


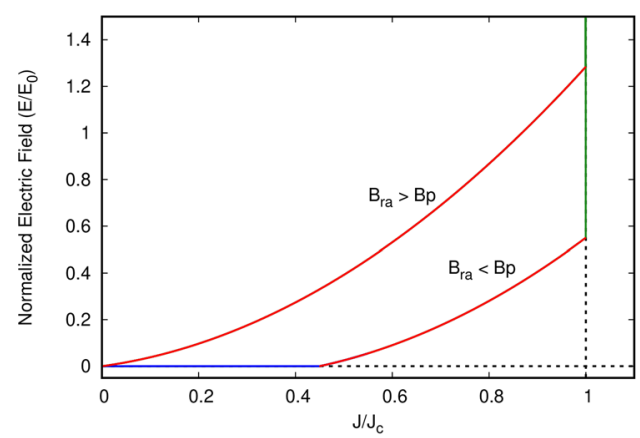

(a)

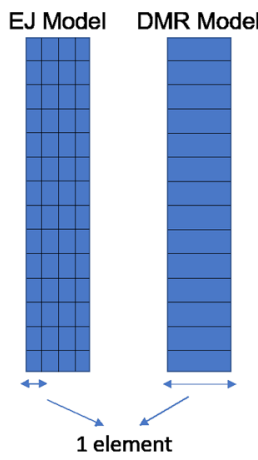

(b)

Figure 1. (a) The $\mathrm{E}(\mathrm{J})$ relation developed for the dynamic-magneto resistance (DMR) model. (b) shows that the DMR model only requires 1 element in thickness mesh for the tape, which makes the calculations faster.

the values 0 or $J_{c}$, but that is not the case for general thin films or 3D shapes, where any $|J| \leq J_{c}$ is allowed ${ }^{51,57-59}$. For numerical purposes of MEMEP, we consider the shunted CSM ${ }^{51,59}$. For benchmarking purposes in "Results and discussion" section, we use the general CSM:

$$
|E|= \begin{cases}0, & \text { if }|J| \leq J_{c} . \\ \text { any, } & \text { if }|J|>J_{c} .\end{cases}
$$

For numerical purposes, we approximate the CSM as a piecewise linear $E(J)$ relation as

$$
E(J)= \begin{cases}0, & \text { if }|J| \leq J_{c} . \\ \rho\left(|J|-J_{c}\right) \frac{J}{|J|}, & \text { if }|J|>J_{c},\end{cases}
$$

where $\rho$ is a very large resistivity, which could have the physical interpretation of a flux-flow resistivity.

Effective constitutive relation from dynamic magneto-resistance. Dynamic magneto-resistance for a slab. For cross-field demagnetization in infinitely long problems, it is very convenient to use the following effective $E(J)$ relation, based on the Dynamic Magneto-Resistance (DMR) ${ }^{39}$. For a slab in the CSM in alternating parallel applied field, $B_{r}$, of amplitude $B_{r a}$, this dynamic magneto-resistance for a given transport current $I$ is $^{39-41,60} R(I)=2 f d l\left[B_{r a}-B_{\text {th }}(I)\right] / I_{c}$, with $B_{t h}, B_{r a}, f, d, l$, and $I_{c}$ being the threshold field, ripple field amplitude, ripple field frequency, tape thickness, tape length, and critical current, respectively. Since we assume a slab in the CSM under a parallel AC field, the threshold field is ${ }^{39} B_{\text {th }}(I)=\mu_{0} J_{c} d\left(1-|I| / I_{c}\right) / 2$, where the critical current, $I_{c}$, follows $I_{c}=w d J_{c}$, and $w$ being the tape width. Then, for a given current $I$, the DC component of the voltage (time average of the voltage in one applied-field cycle) is due to this resistance $V_{R}(I)=R(I) I$. Since the DC electric field, $E_{R}$, is the DC voltage per unit length, $l$, and the average current density in the cross-section, $J_{\mathrm{av}}$, follows $J_{\mathrm{av}}=I /(w d)$, the effective $E(J)$ relation is

$$
E_{R}(J)=E_{0}\left[\frac{B_{r a}}{B_{p}}-\frac{B_{\mathrm{th}}}{B_{p}}\left(\frac{J}{J_{c}}\right)\right] \frac{J}{J_{c}},
$$

where we omit the sub-index " $a v$ " in the current density, for notation simplicity. We wrote the above equation in terms of normalized dimension-less quantities, with $E_{0}$ being $E_{0} \equiv \mu_{0} f d^{2} J_{c}$ and $B_{p}$ being the penetration field of a slab under no transport current, or "parallel penetration field",

$$
B_{p}=\mu_{0} J_{c} d / 2 \text {. }
$$

With this normalization, the threshold magnetic field can be written as

$$
B_{\text {th }}\left(\frac{J}{J_{c}}\right)=B_{p}\left(1-\left|\frac{J}{J_{c}}\right|\right) .
$$

Effective constitutive DMR model. If we divide the thin film into elements across the width with the same thickness as the tape (Fig. 1b, right), we can approximate each element as an individual slab, thanks to their high aspect ratio and negligible mutual magnetic shielding effects of the parallel component of external fields ${ }^{39}$. Then, we can apply the effective $E(J)$ relation of (4) as local for each element, now being $J$ the average current density a the element, and hence it can vary along the tape width. As already shown for a single tape ${ }^{39,45}$, this approach can efficiently model cross-field demagnetization effects in a single tape, as long as it is initially fully saturated due to a perpendicular applied field. Next, we present a more general constitutive relation to also describe demagnetization after partial magnetization or the effect of transverse applied fields simultaneously to the parallel ripple field, as long as their characteristic frequency is much smaller than the ripple field frequency. Although we assume 


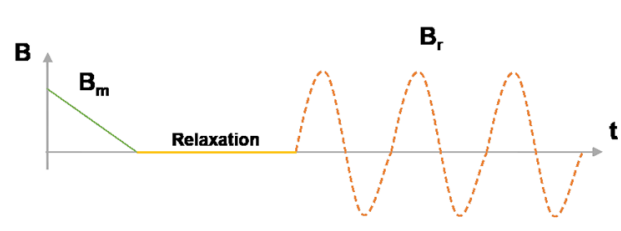

(a)

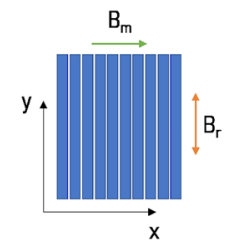

(b)

Figure 2. (a,b) show the demagnetization process of a stack of tapes. First, the magnetizing field $\left(B_{m}\right)$ is applied along the $\mathrm{x}$ direction, ramped down for $100 \mathrm{~s}$, and the tape is let to relax for $900 \mathrm{~s}$. Ripple field $\left(B_{r}\right)$ is then applied along the width of the tape for 30 to millions of cycles.

here constant $J_{\mathcal{c}}$, our effective $E(J)$ relation approach can be easily extended for non-uniform $J_{\mathcal{c}}$ across the tape width or a magnetic-field dependent $J_{c}(\mathbf{B})$.

Combining the CSM $E(J)$ relation of (3) and the effective relation of (4) from DMR, we obtain the effective $E(J)$ relation, being

$$
E(J)= \begin{cases}0 & \text { if } B_{r a} \leq B_{\mathrm{th}}(J) \text { and }|J| \leq J_{c} \\ E_{R}(J) & \text { if } B_{r a} \geq B_{\mathrm{th}}(J) \text { and }|J| \leq J_{c} \\ \rho\left(|J|-J_{c}\right) \frac{J}{|J|}+E_{R}\left(J=J_{c}\right) & \text { if }|J| \geq J_{c}\end{cases}
$$

with $E_{R}\left(J=J_{c}\right)=E_{0} B_{r a} / B_{p}=2 d f B_{r a}$. As seen in Fig. 1a, for $B_{r a}<B_{\text {th }}(J)$ there is no DMR, and hence $E$ vanishes; while for $B_{r a}$ above the parallel penetration field, $B_{p}$, there is non-zero $E$ for any non-zero $J$. We hereby use this DMR model in the MEMEP method for finding effects of cross-field demagnetization of superconducting tape and stacks. The benchmarking and discussion for the same can be seen in the "Results and discussion" section.

Advantages of DMR model. There are two major advantages of DMR model over the general $E(J)$ Power Law model. Firstly, the electric field is calculated for the average current density in the tape thickness for the DMR model. Thus, we need only 1 element in the tape thickness, as is shown in Fig. 1b. In contrast, we need high number of elements (we use up to 200 elements in mesh thickness) for $E(J)$ Power Law model to accurately see the effects of cross field demagnetization on a HTS tape, which makes the simulations more cumbersome and much slower than the DMR model. Also, the electric field is calculated on an average over one whole cycle of ripple field for DMR model, hence we do not need to consider time steps within each cycle, whereas for the $E(J)$ Power Law model, high number of time steps are essential for accurate and detailed analysis of change in current density per time step. Thus, due to these reasons, DMR model is much faster when the simulation is to be run for much higher number of cycles (up to millions!), as we present in this paper.

\section{Modeling configuration}

The cross field demagnetization behavior of superconducting stacks of tapes and bulks have been analyzed in this paper. For tapes, we only take the superconducting layer into account, and hence we assume that the conductivity and permeability of the other layers is negligible (stabilization, substrate, and buffer layers).

The benchmarking of DMR model with $E(J)$ Power Law model has been done on a single tape and a 10 tape stack, with each tape measuring $2 \mu \mathrm{m}$ thickness and $12 \mathrm{~mm}$ width, with $60 \mu \mathrm{m}$ gap between each tape in the stack. The current density used for this case is $1.36 \times 10^{10} \mathrm{~A} / \mathrm{m}^{2}$ at $77 \mathrm{~K}$. The mesh used for $E(J)$ Power Law model is 200 elements in tape thickness and 10 in tape width, with very high accuracy parameters, whereas, for the DMR model, we use only 1 element in thickness and 40 in the tape width. The sample is first magnetized using a field cooling process with magnetizing field of $300 \mathrm{mT}$, ramp down for $100 \mathrm{~s}$, and followed by a relaxation period of $900 \mathrm{~s}$. Later, an alternating sinusoidal cross field of different amplitudes (10-200 mT) is applied at 500 $\mathrm{Hz}$ frequency for 30-100 cycles (Fig. 2a,b).

The stacks used for further analysis are made up of 10 and 100 tapes of $1.5 \mu \mathrm{m}$ thickness and $40 \mathrm{~mm}$ width, with gap as $100 \mu \mathrm{m}$ between tapes. This configuration corresponds to the widest commercially available tape, which is very promising to trap high magnetic fields ${ }^{46}$. The current density used for this analysis is $5.78 \times 10^{10} \mathrm{~A} /$ $\mathrm{m}^{2}$, which roughly corresponds to the values of American Superconductor tape at $30 \mathrm{~K}$. The initial magnetizing field is $5 \mathrm{~T}$ for 10 tape stack, and $10 \mathrm{~T}$ for 100 tape stack, to ensure the complete penetration and magnetization of the stacks. We also consider that various amplitudes of cross field (15-200 mT) are applied at $2400 \mathrm{~Hz}$ frequency. The reason for using such high frequencies in both cases is to simulate the environment of a superconducting motor, where background ripple fields are at frequencies of this order of magnitude due to the present harmonics. The modeling results in this article used a computer with an Intel i7-7700 processor of 8 logical cores, 16 GB RAM, and Linux operating system.

\section{Results and discussion}

Benchmark of the DMR model. Firstly, the current density maps for a single tape using critical state model and DMR model can be observed in Fig. 3. In both cases the tape is fully magnetized at the end of the field cooling magnetization, and we also do not see any relaxation after magnetization since we have used Critical State Model in the $E(J)$ relations for DMR model. The tape is seen to show considerable demagnetization after 

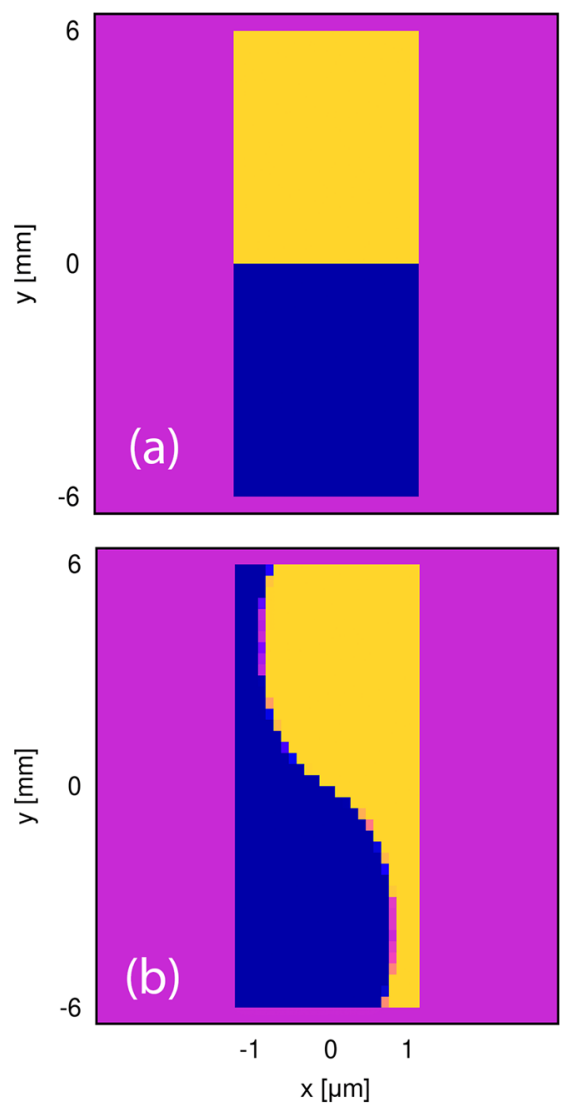

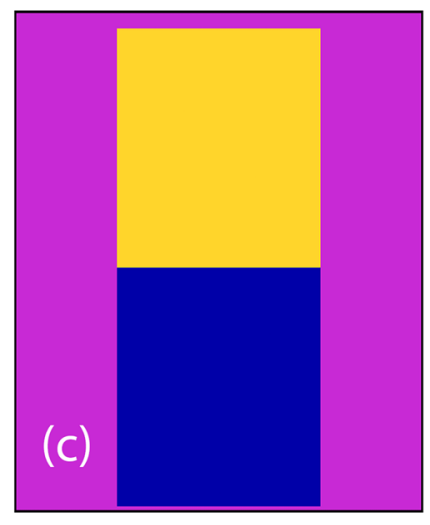

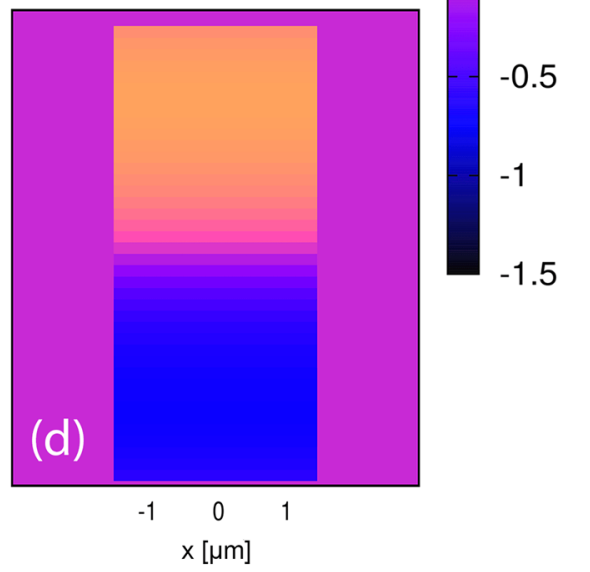

1.5

1

0.5

$0 \stackrel{0}{\supset}$

Figure 3. Current density profiles for a single tape under critical state model (a,b) and DMR model (c,d) after Field Cool Magnetization and Relaxation $(\mathbf{a}, \mathbf{c})$, and 30 cycles of demagnetizing ripple field $(\mathbf{b}, \mathbf{d})$. Single element is used in the thickness for DMR model. Thickness is also artificially expanded for better analysis.

application of 30 cycles of ripple field, and we get the typical S shape of the current profile here. For DMR model, the change is only seen in the width of the tape, since we use only single element in the tape thickness (Fig. 3d), in contrast to Critical State Model, where we have up to 24 elements in the tape thickness (Fig. 3b).

Next, we compare DMR model with $E(J)$ Power Law model and approximated Critical State Model (CSM) for a single tape in Fig. 4a,b. It can be seen from Fig. 4a that there is no relaxation for CSM model and DMR model, in continuation with our discussion regarding current density profiles. Fig. $4 \mathrm{~b}$ shows very good agreement between all three cases regarding trapped field behavior during ripple field application. In Fig. 4c, a 10 tape stack at 77 $\mathrm{K}$ is compared for DMR and $E(J)$ Power Law model for 100 cycles of ripple field. The current density profile for a 10 tape stack after 100 cycles of demagnetization is shown in Fig. 4d. Here again the DMR model agrees very accurately with the $E(J)$ Power Law model. The time taken to simulate this case for $E(J)$ Power Law model was up to 1-2 months, depending on the ripple field amplitude. In contrast to this, DMR model took less than $2 \mathrm{~min}$ to achieve the same results, which validates the high accuracy, speed, and strength of this model. It is important to state here that for more detailed analyses, such as calculating absolute current densities at each time step and at each section of the tape, $E(J)$ Power Law model should be used. Now, as the DMR model is successfully benchmarked, we move forward to simulate demagnetization for thicker stacks and for high number of cycles.

DMR results for high number of cycles. Firstly, we calculate trapped field behavior for a 10 tape stack at $30 \mathrm{~K}$, with each tape size being $1.5 \mu \mathrm{m} \times 40 \mathrm{~mm}$, for 100,000 ripple field cycles (see Fig. 5). It is seen that there is more demagnetization at high ripple field amplitudes, which is due to the dynamic magneto-resistance that exists above the threshold field of the tape ${ }^{39}$. The trapped field goes to zero for ripple field amplitudes above the parallel penetration field of one tape (Eq. (5)), which is around $55 \mathrm{mT}$ for this case. However, at higher number of cycles, we also see that the trapped field reaches an asymptotic value for ripple field amplitudes below the parallel penetration field. This non-zero asymptotic value appears because for ripple fields below the penetration field, the dynamic magneto-resistance vanishes below a certain current density (Fig. 1), and hence the current density does not further decay when it reaches this threshold value. With decreasing the ripple field amplitude, the threshold current density increases, also increasing the asymptotic trapped field. This behavior qualitatively agrees with Brandt's results for a single tape ${ }^{39}$.

To test the robustness of the DMR model, we also find results for a 100 tape stack for up to 2 million cycles of applied ripple field. The current profiles for this case are shown in Fig. 6. It can be seen here that in the case of $30 \mathrm{mT}$, the current density in the stack is only reduced by around half of its original value at relaxation, and 

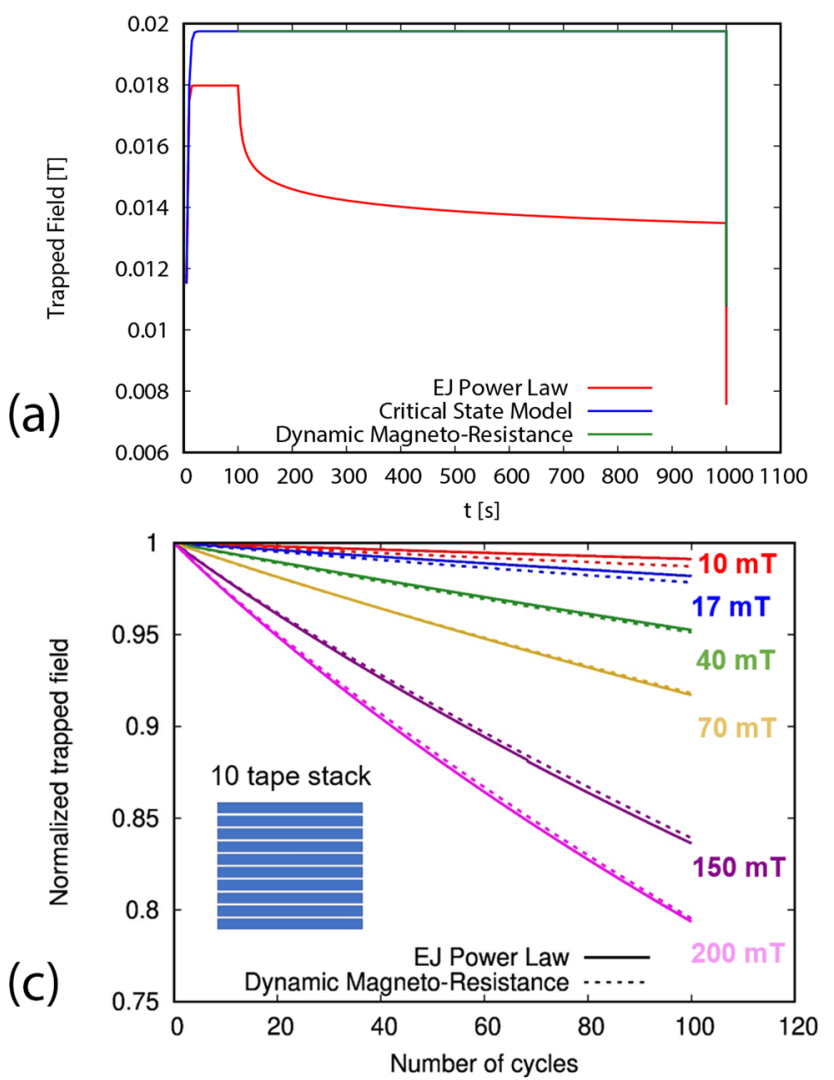
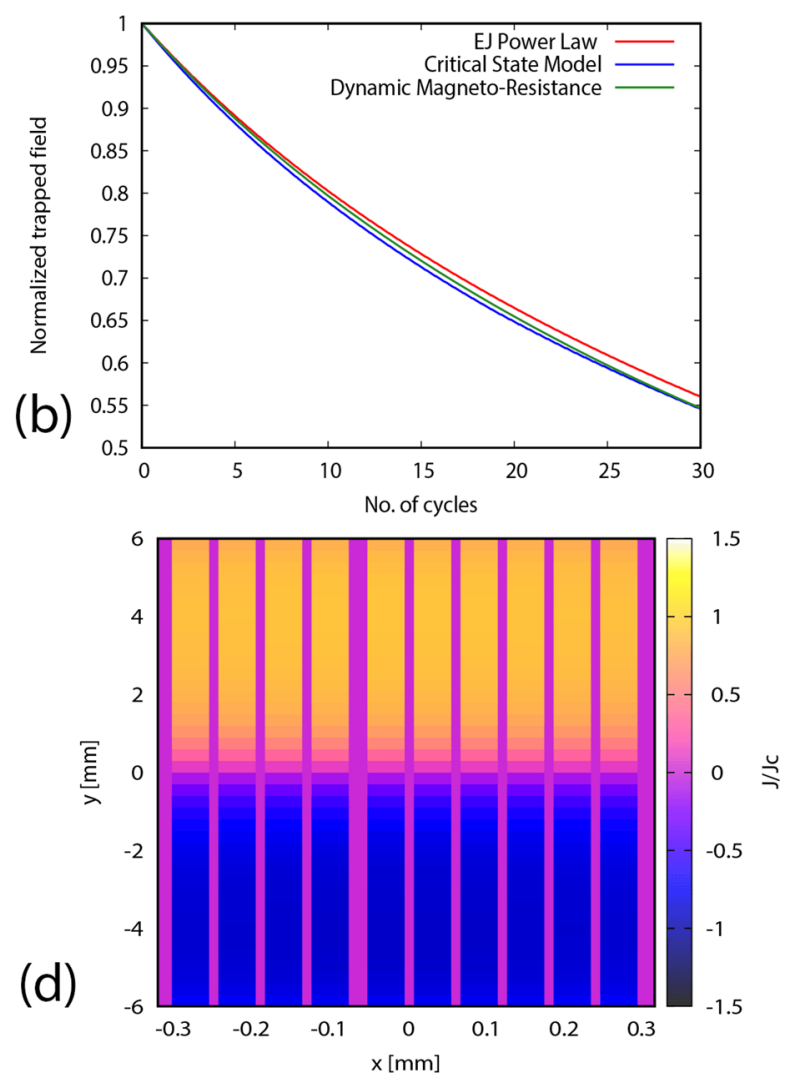

Figure 4. Benchmark of DMR model, and approximated Critical State Model, with $E(J)$ Power Law model for a single tape for (a) the whole trapped field evolution, and (b) the demagnetization process, at 30 cycles of ripple field and $200 \mathrm{mT}$ at high temperature $(77 \mathrm{~K})$. A 10 tape stack is compared in (c) for higher number of cycles. DMR model shows good agreement with $E(J)$ power law model for HTS single tape and stack and is much faster. (d) Current density profile for the stack after 100 cycles of demagnetization at $200 \mathrm{mT}$ using DMR model. Thickness is artificially expanded for better visibility and considerable demagnetization is observed in the stack.

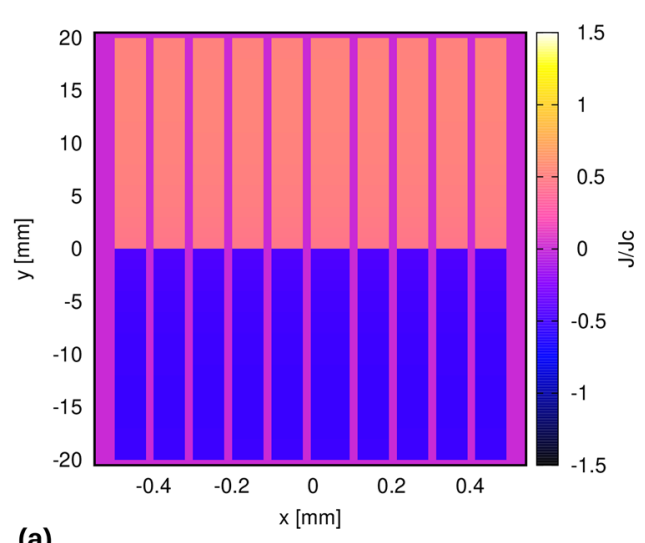

(a)

Figure 5. Demagnetization behavior of a stack of 10 tapes for high number of cycles $(100,000)$ at low temperature $(30 \mathrm{~K})$. (a) shows the current profile after 100,000 cycles for $30 \mathrm{mT}$ ripple field. (b) The trapped field reaches an asymptotic value for field amplitudes below the tape's penetration field (around $55 \mathrm{mT}$ ).

hence the magnetization is also half (Fig. 6). As for the case of 10 tapes, the reason for this is that at fields below penetration field of the stack (around $55 \mathrm{mT}$ ), the trapped field reaches at an asymptotic value, and thus the stack retains considerable amount of magnetization indefinitely. An important point to note here is that the relative retention of the trapped field is independent on the number of tapes in the stack, being the same for 10 and 100 tapes. The reason is that the asymptotic value of the current density only depends on the ripple field amplitude and the parallel penetration field of the tape. Increasing the number of tapes only increases the number of cycles necessary to reach the asymptotic value. 

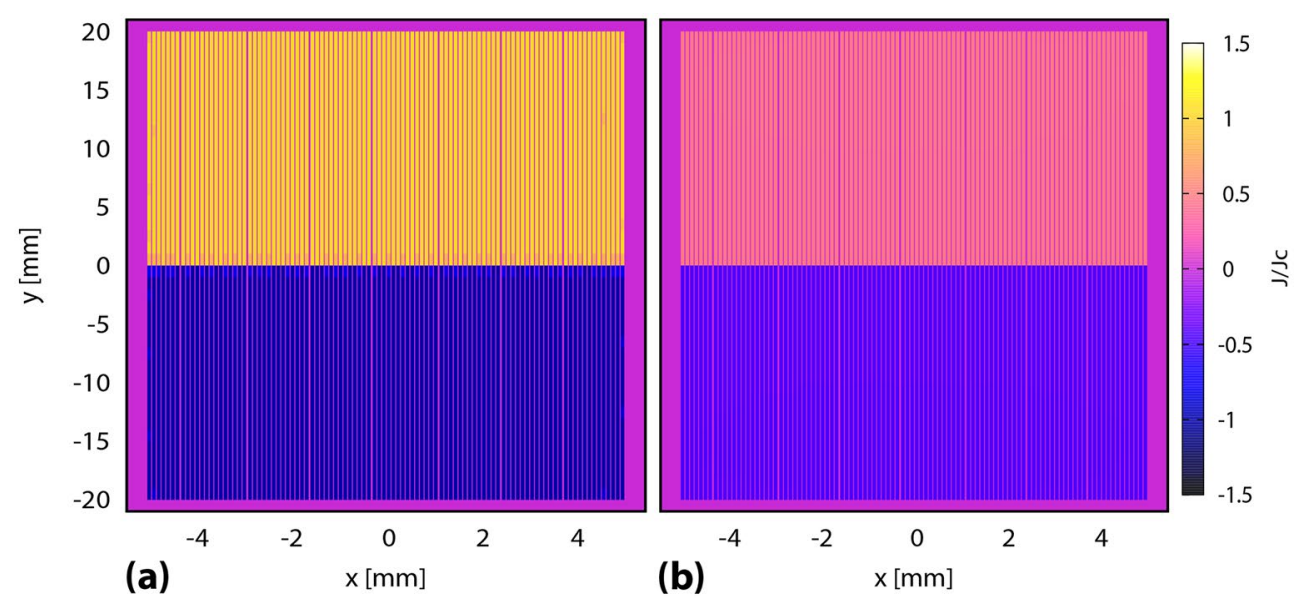

Figure 6. Current profiles of a stack of 100 tapes after (a) magnetization and relaxation period, and (b) 2 million cycles of ripple field at $30 \mathrm{mT}$ at low temperature $(30 \mathrm{~K})$. Thickness of the tapes is expanded artificially for better visibility of results. At $30 \mathrm{mT}$, the stack retains almost half of its original magnetization indefinitely.
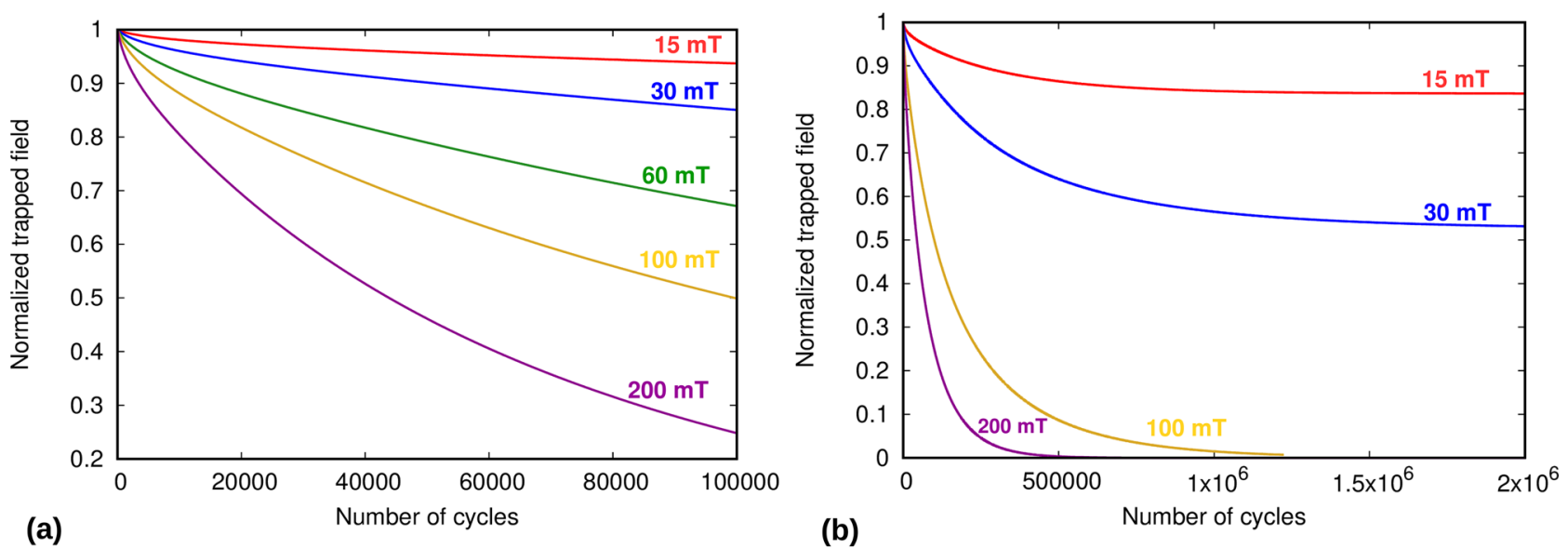

Figure 7. Demagnetization behavior of a stack of 100 tapes for (a) 100 thousand cycles, and (b) 2 million cycles at low temperature $(30 \mathrm{~K})$. The stack reaches an asymptotic value at ripple fields below penetration field of a tape $(55 \mathrm{mT})$.

Figure 7 shows that it is essential to have analysis of very high number of cycles to get a complete understanding of the process. From high number of cycles, we can get answers to questions, such as when exactly the stack will fully demagnetize or when will it arrive at an asymptotic value. For applications such as fully superconducting electric motors for aviation propulsion systems, this is a very important analysis as it can tell us about the eventual power output of the motor after it starts to get demagnetized, and by what time the motor will reach at this range of power. For this case, it is important to note here that two million cycles for such a high frequency $(2400 \mathrm{~Hz})$ corresponds to only about $14 \mathrm{~min}$. Thus, for example, the magnetization of a superconducting stack with these parameters, in a practical environment, will reduce to approximately $53 \%$ in just around $7 \mathrm{~min}$ if the ripple field amplitude is kept to about $30 \mathrm{mT}$. For lower frequencies, the decay time will be longer. Thus, the primary goal of a motor designer can be to keep the ripple field amplitude as low as possible, and use tapes with the highest available critical current per unit tape width (at the opeartional temperature and background magnetic field) to avoid as much cross field demagnetization as possible. Frequency reduction and increase of number of tapes is secondary, since it only increases the transient decay time.

The time taken for the simulation of 100-tape stack for 2 million cycles is only around 2 days, which affirms to the potential capability of the DMR model. Moreover, our modeling tool enables to run several calculations in parallel, further reducing the average computing time.

Comparison of bulk vs stack. Next, we discuss an interesting comparison of a superconducting stack and bulk. For this purpose, we use a 10 tape stack with the same parameters as in previous section (for $30 \mathrm{~K}$ ). The dimensions of the equivalent bulk used here are the same as the stack; as well as the engineering critical current density (or total current density per unit overall volume), being 

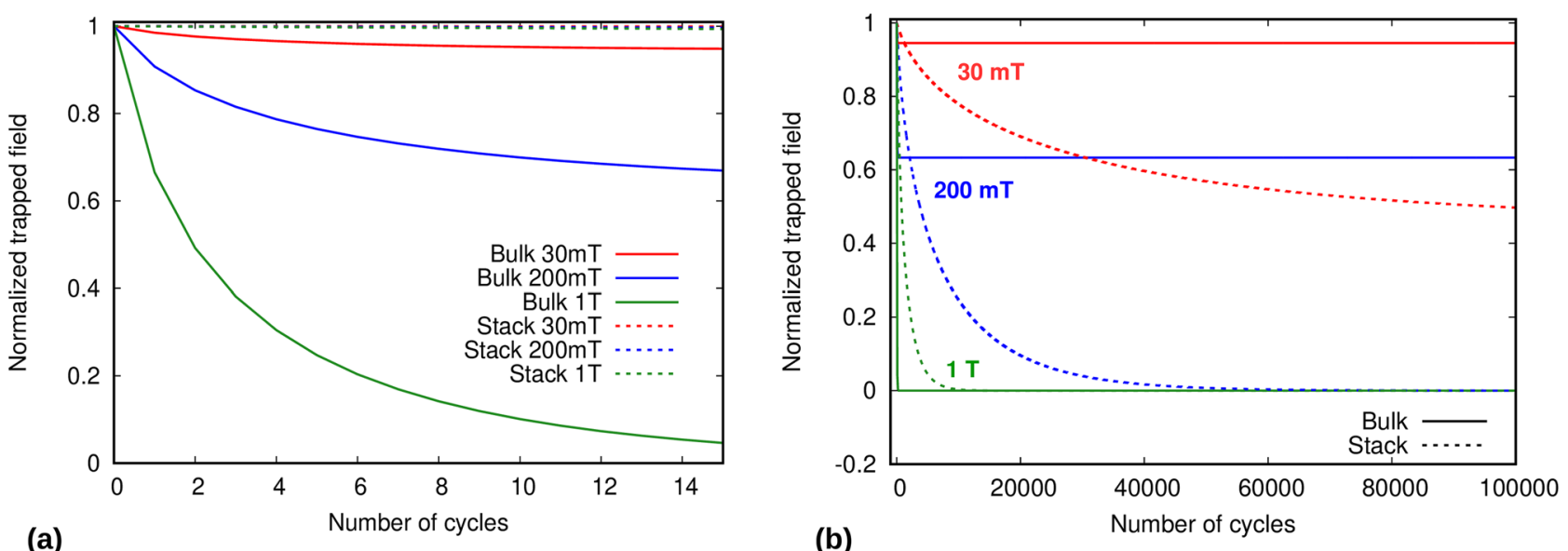

Figure 8. Comparison of the demagnetization behavior of a bulk and stack for (a) low number of cycles, and (b) high number of cycles.

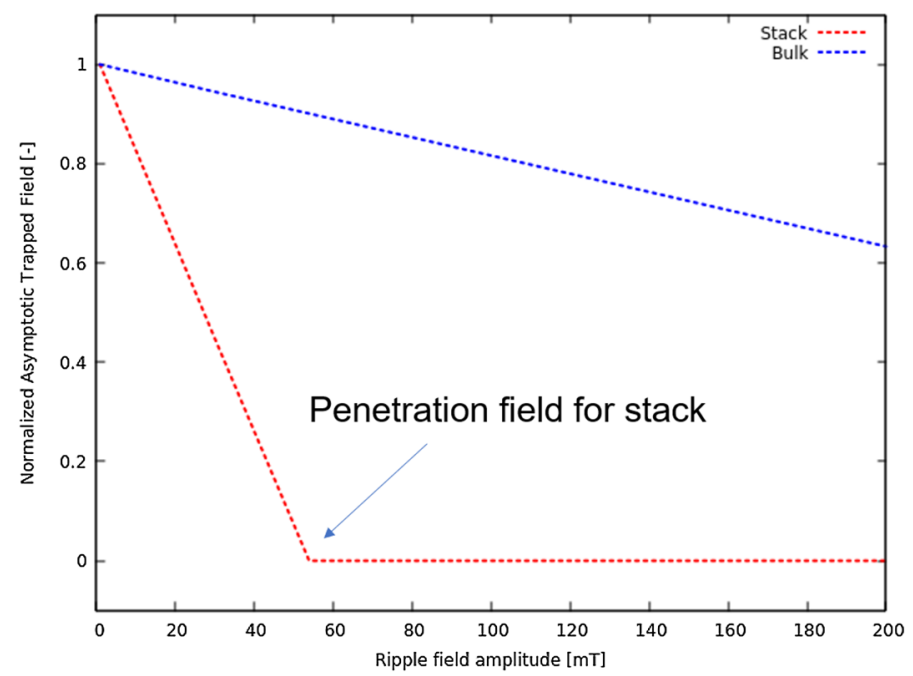

Figure 9. Asymptotic field values for bulk and stack. Bulk has higher asymptotic values as compared to a same size stack and equal engineering critical current density.

$$
J_{c e}=J_{c} n_{t} d \frac{1}{D}
$$

where, $n_{t}, d$, and $D$ are number of tapes in the stack, superconductor layer thickness of a tape in the stack, and total height of the stack, respectively.

The comparison of the bulk and stack can be seen in the Fig. 8. The parts (a) and (b) show a striking contrast between both the bulk's and the stack's trapped field behavior during demagnetization. For low number of cycles, we see that the stacks are much better than the bulks at all ripple field amplitudes, with losing almost no magnetization, whereas the bulks show a very fast transient decay for the same cases, and even going to zero at very high ripple field amplitudes. Figure $8 \mathrm{~b}$ shows that although the bulks experience a very fast initial decrease of magnetization, the permanent asymptotic values are much higher than for the stacks. The reason for such a behavior is that bulks have much higher parallel penetration field than stacks, which enables them to sustain assimptotic trapped fields for higher ripple field amplitudes (Fig. 9). Thus, for high number of cycles, the bulks are better, as their stable asymptotic field values are much higher than the stack. For low number of cycles, the stacks are much better than the bulks because stacks require orders of magnitude more cycles to demagnetize. Therefore, for applications with high amplitudes of ripple field and low number of cycles (or very low frequencies), HTS stacks are preferred. In addition, they also have some other benefits over bulks, such as flexibility and ease of construction. However, for applications involving high ripple fields amplitudes and frequencies (or long run times), bulks should be used, because of their higher asymptotic values as compared to stacks. 


\section{Conclusion}

Cross field demagnetization of stacks is a major issue for a fully superconducting motors. The model based on dynamic magneto-resistance (DMR Model) can very accurately predict this situation for high number of ripple field cycles (up to millions), and very thick stacks, at a very high speed as compared to the conventional numerical models using $E(J)$ Power Law. The results show that, for high number of cycles, superconducting stacks reach a non-zero static asymptotic value, if the applied ripple field is below the parallel penetration field of a tape. This asymptotic value depends on the tape properties only, and hence it does not increase with the number of tapes in the stack. For bulks, the asymptotic value is much higher than for stacks. We also see that bulks reach asymptotic values much faster than the stack. In contrast, stacks do not lose much magnetization if the runtime of the application involve low number of cycles, even if the ripple field amplitudes are high. In motors for aviation, where ripple fields can range from few hundreds of Hertz to kilo-Hertz, the asymptotic value could be reached in times much shorter than the flight-times, being bulks more reliable for these applications, unless the ripple fields in the stack are strongly reduced ${ }^{61}$.

Received: 18 June 2020; Accepted: 22 October 2020

Published online: 06 November 2020

\section{References}

1. Abrahamsen, A. et al. Superconducting wind turbine generators. Supercond. Sci. Technol. 23, 034019 (2010).

2. Lloberas, J., Sumper, A., Sanmarti, M. \& Granados, X. A review of high temperature superconductors for offshore wind power synchronous generators. Renew. Sustain. Energy Rev. 38, 404-414 (2014).

3. Bergen, A. et al. Design and in-field testing of the worlds first ReBCO rotor for a $3.6 \mathrm{MW}$ wind generator. Supercond. Sci. Technol. 32, 125006 (2019).

4. American Superconductors. Superconducting motors. in Azo Materials (2001). www.azom.com/article.aspx?ArticleID=949.

5. Snitchler, G., Gamble, B. \& Kalsi, S. The performance of a $5 \mathrm{mw}$ high temperature superconductor ship propulsion motor. IEEE Trans. Appl. Supercond. 15, 2206-2209 (2005).

6. Gamble, B., Snitchler, G. \& MacDonald, T. Full power test of a $36.5 \mathrm{mw}$ hts propulsion motor. IEEE Trans. Appl. Supercond. 21(3), 1083-1088 (2011).

7. Zhou, D. et al. An overview of rotating machine systems with high-temperature bulk superconductors. Supercond. Sci. Technol. 25, 103001 (2012).

8. Haran, K. et al. High power density superconducting rotating machines-development status and technology roadmap. Supercond. Sci. Technol. 30, 123002 (2017).

9. Nam, G., Sung, H., Kim, C., Park, M. \& Yu, I.-K. Design and characteristic analysis of a 1 MW superconducting motor for ship propulsions. IEEE Trans. Appl. Supercond. 29, 1-5 (2019).

10. Kusada, S. et al. The project overview of the HTS magnet for superconducting maglev. IEEE Trans. Appl. Supercond. 17, 2111-2116 (2007).

11. Uno, M. Chuo shinkansen project using superconducting maglev system. Jpn. Railway Transp. Rev. 68, 14-25 (2016).

12. Zheng, L. \& Jin, J. Studying the force characteristics of a high temperature superconducting linear synchronous motor. J. Appl. Phys. 110, 043915 (2011).

13. Park, C.-B. Performance comparison between the normal-conducting magnet and the superconducting magnet in LSM for highspeed propulsion. IEEE Trans. Magn. 53, 8204106 (2017).

14. Gong, T. et al. Calculation and optimization of propulsion force of a real-scale REBCO magnet for EDS train. IEEE Trans. Appl. Supercond. 29, 3602606 (2019).

15. Choi, S. et al. Sub-sonic linear synchronous motors using superconducting magnets for the hyperloop. Energies 12, 4611 (2019).

16. Oswald. ASuMED-Advanced Superconducting Motor Experimental Demonstrator. H2020 Project (2017). www.asumed.oswal d.de.

17. Boll, M. et al. A holistic system approach for short range passenger aircraft with cryogenic propulsion system. Supercond. Sci. Technol. 33, $044014(2020)$.

18. Sugouchi, R. et al. Conceptual design and electromagnetic analysis of $2 \mathrm{MW}$ fully superconducting synchronous motors with superconducting magnetic shields for turbo-electric propulsion system. IEEE Trans. Appl. Supercond. 30, 3601905 (2020).

19. Grilli, F. et al. Superconducting motors for aircraft propulsion: The advanced superconducting motor experimental demonstrator project. J. Phys. Conf. Ser.https://doi.org/10.1088/1742-6596/1590/1/012051 (2020).

20. Durrell, J. H. et al. A trapped field of $17.6 \mathrm{~T}$ in melt-processed, bulk Gd-Ba-Cu-O reinforced with shrink-fit steel. Supercond. Sci. Technol. 27, 082001 (2014).

21. Patel, A. et al. A trapped field of $17.7 \mathrm{~T}$ in a stack of high temperature superconducting tape. Supercond. Sci. Technol. 31, 09LT01 (2018).

22. Sotelo, G. G., Sass, F., Carrera, M., Lopez-Lopez, J. \& Granados, X. Proposal of a novel design for linear superconducting motor using $2 \mathrm{~g}$ tape stacks. IEEE Trans. Ind. Electron. 65, 7477 (2018).

23. Patel, A., Climente-Alarcon, V., Baskys, A. \& Glowacki, B. A. Design considerations for fully superconducting synchronous motors aimed at future electric aircraft. IEEE Int. Conf. Electr. Syst.https://doi.org/10.1109/ESARS-ITEC.2018.8607734 (2018).

24. Smara, A. et al. An experimental assessment of rotor superconducting stack demagnetization in a liquid nitrogen environment. Supercond. Sci. Technol. 32, 085009 (2019).

25. Yanamoto, T., Izumi, M., Murase, Y. \& Oryu, T. Loss analysis of a 3-MW high-temperature superconducting ship propulsion motor. IEEE Trans. Appl. Supercond. 28, 1-5 (2018).

26. Werfel, F. N. et al. Superconductor bearings, flywheels and transportation. Supercond. Sci. Technol. 25, 014007 (2012).

27. Sotelo, G. G. et al. A full scale superconducting magnetic levitation (MagLev) vehicle operational line. IEEE Trans. Appl. Supercond. 25, 3601005 (2015).

28. Deng, Z. et al. A high-temperature superconducting maglev ring test line developed in Chengdu, China. IEEE Trans. Appl. Supercond. 26, $3602408(2016)$

29. Sass, F., Nogueira Dias, D. H., Gonçalves Sotelo, G. \& de Andrade, R. Superconducting levitation using coated conductors. IEEE Trans. Appl. Supercond. 23, 3600905 (2013).

30. Patel, A. et al. Magnetic levitation using a stack of high temperature superconducting tape annuli. Supercond. Sci. Technol. 30, 024007 (2017).

31. Sass, F., Dias, D., Sotelo, G. \& de Andrade Junior, R. Superconducting magnetic bearings with bulks and 2G HTS stacks: Comparison between simulations using $\mathrm{H}$ and AV formulations with measurements. Supercond. Sci. Technol. 31, 025006 (2018).

32. Liu, K. et al. Experiment and simulation of superconducting magnetic levitation with REBCO coated conductor stacks. Supercond. Sci. Technol. 31, 015013 (2018). 
33. Hahn, S. et al. Trapped field characteristics of stacked ybco thin plates for compact NMR magnets: Spatial field distribution and temporal stability. IEEE Trans. Appl. Supercond. 20, 1037-1040 (2010).

34. Patel, A., Hopkins, S. \& Glowacki, B. Trapped fields up to $2 \mathrm{~T}$ in a $12 \mathrm{~mm}$ square stack of commercial superconducting tape using pulsed field magnetization. Supercond. Sci. Technol. 26, 032001 (2013).

35. Campbell, A. M. Solving the critical state using flux line properties. Supercond. Sci. Technol. 27, 124006 (2014).

36. Baghdadi, M., Ruiz, H. S. \& Coombs, T. A. Crossed-magnetic-field experiments on stacked second generation superconducting tapes: Reduction of the demagnetizing effects. Appl. Phys. Lett. 104, 232602 (2018).

37. Baghdadi, M., Ruiz, H. S. \& Coombs, T. A. Nature of the low magnetization decay on stacks of second generation superconducting tapes under crossed and rotating magnetic field experiments. Sci. Rep. 8, 1342 (2018).

38. Kapolka, M. et al. Cross-field demagnetization of stacks of tapes: 3D modelling and measurements. Supercond. Sci. Technol. 33, 044019 (2020).

39. Brandt, E. \& Mikitik, G. Why an AC magnetic field shifts the irreversibility line in type-II superconductors. Phys. Rev. Lett.https ://doi.org/10.1103/PhysRevLett.89.027002 (2002).

40. Ogasawara, T. et al. Alternating field losses in superconducting wires carrying dc transport currents: Part 1 single core conductors. Cryogenics 19, 736-740 (1979).

41. Jiang, Z., Toyomoto, R., Amemiya, N., Zhang, X. \& Bumby, C. Dynamic resistance of a high-Tc coated conductor wire in a perpendicular magnetic field at 77 K. Supercond. Sci. Technol.https://doi.org/10.1088/1361-6668/aa54e5/meta (2017).

42. Talantsev, E. F. et al. The onset of dissipation in high-temperature superconductors: Magnetic hysteresis and field dependence. Sci. Rep. 8, 14463 (2018).

43. Mikitik, G. \& Brandt, E. Theory of the longitudinal vortex-shaking effect in superconducting strips. Phys. Rev. B 67, 104511 (2003).

44. Campbell, A. M. et al. Demagnetisation by crossed fields in superconductors. Supercond. Sci. Technol. 30, 034005 (2017).

45. Dadhich, A., Pardo, E. \& Kapolka, M. Time constant of the transverse-field demagnetization of superconducting stacks of tapes. Supercond. Sci. Technol.https://doi.org/10.1088/1361-6668/ab877b/pdf (2020).

46. Climente-Alarcon, V., Patel, A., Baskys, A. \& Glowacki, B. A. Design considerations for electric motors using stacks of high temperature superconducting tape as permanent magnets. Conf. Ser. Mater. Sci. Eng. 502, 02182 (2019).

47. Baskys, A., Patel, A. \& Glowacki, B. Measurements of crossed-field demagnetization rate of trapped field magnets at high frequencies and below 77k. Supercond. Sci. Technol.https://doi.org/10.1088/1361-6668/aabf32 (2018).

48. Vanderbemden, P. et al. Behavior of bulk high-temperature superconductors of finite thickness subjected to crossed magnetic fields: Experiment and model. Phys. Rev. B 75, 174515 (2007).

49. Kapolka, M. et al. Three-dimensional modeling of the magnetization of superconducting rectangular-based bulks and tape stacks. IEEE Trans. Appl. Supercond. 28, 8201206 (2018).

50. Srpcic, J. et al. Penetration depth of shielding currents due to crossed magnetic fields in bulk RE-Ba-Cu-O superconductors. Supercond. Sci. Technol. 32, 035010 (2019).

51. Srpcic, J. et al. Demagnetization study of pulse-field magnetized bulk superconductors. IEEE Trans. Appl. Supercond. 28, 6801305 (2018).

52. Pardo, E., Šouc, J. \& Frolek, L. Electromagnetic modelling of superconductors with a smooth current-voltage relation: Variational principle and coils from a few turns to large magnets. Supercond. Sci. Technol. 28, 044003 (2015).

53. Pardo, E. \& Kapolka, M. 3D computation of non-linear eddy currents: Variational method and superconducting cubic bulk. J. Comput. Phys. 344, 339-363 (2017).

54. Grilli, F. \& Pardo, E. Simulation of ac loss in roebel coated conductor cables. Supercond. Sci. Technol. 23, 115018 (2010).

55. Pardo, E. \& Grilli, F. Numerical simulations of the angular dependence of magnetization AC losses: Coated conductors, roebel cables and double pancake coils. Supercond. Sci. Technol. 25, 014008 (2012).

56. Prigozhin, L. Analysis of critical-state problems in type-II superconductivity. IEEE Trans. Appl. Supercond. 7, 3866-3873 (1997).

57. Anderson, P. Theory of flux creep in hard superconductors. Phys. Rev. Lett. 9, 309 (1962).

58. Prigozhin, L. On the bean critical-state model in superconductivity. Eur. J. Appl. Math. 7, 237-247 (1996).

59. Bossavit, A. Numerical modelling of superconductors in three dimensions: A model and a finite element method. IEEE Xplore 30(5), 3363-3366 (1994)

60. Ogasawara, T., Yasukochi, K., Nose, S. \& Sekizawa, H. Effective resistance of current-carrying superconducting wire in oscillating magnetic fields 1: Single core composite conductor. Cryogenics 16, 33-38 (1976).

61. Climente-Alarcon, V. et al. Magnetization and losses for an improved architecture of trapped-flux superconducting rotor. J. Propul. Power 36, 101-108. https://doi.org/10.2514/1.B37709 (2020).

\section{Acknowledgements}

The authors acknowledge the financial support by the European Union's Horizon 2020 research innovation program under grant agreement No 7231119 (ASuMED consortium), as well as from the Grant Agency of the Ministry of Education of the Slovak Republic and the Slovak Academy of Sciences (VEGA) under contract No. 2/0097/18 and the Slovak Research and Development Agency (APVV) under contract No. APVV-19-0536.

\section{Author contributions}

Both A.D. and E.P. wrote the article; E.P. conceived the numerical simulations background and physical model; A.D. further developed the simulation software and performed the numerical simulations; E.P. concluded the analysis and interpretation; A.D. prepared all figures.

\section{Competing interests}

The authors declare no competing interests.

\section{Additional information}

Correspondence and requests for materials should be addressed to E.P.

Reprints and permissions information is available at www.nature.com/reprints.

Publisher's note Springer Nature remains neutral with regard to jurisdictional claims in published maps and institutional affiliations. 
(c) (i) Open Access This article is licensed under a Creative Commons Attribution 4.0 International cc) License, which permits use, sharing, adaptation, distribution and reproduction in any medium or format, as long as you give appropriate credit to the original author(s) and the source, provide a link to the Creative Commons licence, and indicate if changes were made. The images or other third party material in this article are included in the article's Creative Commons licence, unless indicated otherwise in a credit line to the material. If material is not included in the article's Creative Commons licence and your intended use is not permitted by statutory regulation or exceeds the permitted use, you will need to obtain permission directly from the copyright holder. To view a copy of this licence, visit http://creativecommons.org/licenses/by/4.0/.

(C) The Author(s) 2020 